\begin{tabular}{|c|l|l|l|l|l|}
\hline $\begin{array}{l}\text { Abrechnungsziffern und Multiplikatoren bei Anfragen privater } \\
\text { Versicherungen nach GOÄ }\end{array}$ & Tabelle 1 \\
\hline GOÄ-Nr. & Leistung & Punkte & $\begin{array}{l}\text { x 1,0- } \\
\text { fach }\end{array}$ & $\begin{array}{l}\text { x 2,3- } \\
\text { fach }\end{array}$ & $\begin{array}{l}\text { x 3,5- } \\
\text { fach }\end{array}$ \\
\hline 70 & Kurze Bescheinigung & 40 & $2,33 €$ & $5,36 €$ & $8,16 €$ \\
\hline 75 & Arztbericht & 130 & $7,58 €$ & $17,43 €$ & $26,52 €$ \\
\hline 80 & Gutachterliche Stellungnahme & 300 & $17,49 €$ & $24,13 €$ & $36,72 €$ \\
\hline 85 & $\begin{array}{l}\text { Ausführliches Gutachten, je } \\
\text { angefangene Stunde }\end{array}$ & 500 & $29,14 €$ & $67,03 €$ & $102,00 €$ \\
\hline 95 & Schreibgebühr pro Seite & 60 & $3,05 €$ & & \\
\hline 96 & Duplikat pro Seite & 3 & $0,17 €$ & & \\
\hline & & & & \\
\hline
\end{tabular}

\section{Pauschale für die fachärztliche Grundversorgung}

Zum 1. Oktober 2013 zündet die erste Stufe zur Weiterentwicklung des FacharztEBM mit der Einführung der "Pauschale für die fachärztliche Grundversorgung".

$\mathrm{N}$ ach vielen Diskussionen wurden jetzt die Fachgruppen, für die die „Pauschale für die fachärztliche Grundversorgung" (PFG) zutreffend ist, durch den Bewertungsausschuss festgelegt, ebenso die Punktbewertung für die einzelnen Fachgruppen. Der grundsätzliche Leistungsinhalt ist für alle Fachgruppen derselbe, lediglich die Punktbewertung differiert.

Es handelt sich um einen Zuschlag zu den abrechenbaren Grundpauschalen der einzelnen Fachgruppen, der lediglich von für die vertragsärztliche Versorgung zugelassenen Vertragsärzten und MVZ abgerechnet werden kann. Auch die Fachgruppen der neurologischen und psychiatrischen Versorgung kommen in den Genuss dieser Pauschale, allerdings mit unterschiedlicher Bewertung (Tabelle 1).

Die PFG kann in allen Behandlungsfällen angesetzt werden, in denen lediglich Leistungen abgerechnet werden, die der fachärztlichen Grundversorgung ent- rarvereinbarung kommt aber nur dann zustande, wenn der Versicherer die Honoraranfrage bestätigt und die Zahlung zusagt. Am einfachsten ist dann die Angabe eines Stundensatzes von beispielsweise $180 €$ mit der voraussichtlichen Bearbeitungszeit von etwa 30 Minuten. Natürlich sind daneben auch Schreibgebühren, Portokosten, Kopierkosten und sonstige Auslagen berechenbar.

Nicht unerwähnt darf bleiben, dass bei entsprechenden Umsatzgrößen umsatzsteuerpflichtiger Leistungen (jenseits der Kleinunternehmerregelung) immer auch zusätzlich die Mehrwertsteuer in Rechnung gestellt werden muss. Auch darauf sollte dann bei der Honoraranfrage hingewiesen werden.

Dr. med. Heiner Pasch E-Mail:drpasch@t-online.de

\section{Aufnahme einer Nr. 8 in die Allgemeinen Bestimmungen 4.3 (zum 1. Oktober 2013)}

\section{EBM, Allgemeine Bestimmungen}

4.3.8 "Fachärztliche Grundversorgung": In Behandlungsfällen in denen ausschließlich Leistungen erbracht werden, die gemäß der Kennzeichnung des Anhangs 3 des EBM der fachärztlichen Grundversorgung zugerechnet werden, können als Zuschlag zu den entsprechenden Grundpauschalen die arztgruppenspezifischen Leistungen für die fachärztliche Grundversorgung der einzelnen Kapitel berechnet werden...

\begin{tabular}{|l|l|l|}
$\begin{array}{l}\text { Pauschalen für die fachärztliche Grundversorgung im Bereich der } \\
\text { neurologisch-psychiatrisch-psychotherapeutischen Versorgung }\end{array}$ & Tabelle 1 \\
\hline EBM-Nr. & Leistung & Bewertung \\
\hline 16215 & $\begin{array}{l}\text { Zuschlag für die neurologische Grundversorgung gemäß Allge- } \\
\text { meiner Bestimmung } 4.3 .8 \text { zu den Gebührenordnungspositio- } \\
\text { nen } 16210 \text { bis } 16212\end{array}$ & 35 Punkte / 3,50€ \\
\hline 21218 & $\ldots$ psychiatrische und nervenheilkundliche ... 21210 bis 21215 & 35 Punkte / 3,50€ \\
\hline 22216 & $\ldots$ psychotherapeutisch-medizinische ... 22210 bis 22212 & 159 Punkte / 15,90€ \\
\hline 23216 & $\ldots$ psychotherapeutische ... 23210 bis 23212 und 23214 & 159 Punkte / 15,90€
\end{tabular}

sprechen. Eine Liste der Leistungen des EBM, die nicht Teil der fachärztlichen Grundversorgung sind, findet sich im Anhang 3 zum EBM. Diese Leistungen sind mit einem „* " markiert. Neben fachgruppenspezifischen Leistungen sind hier auch eine Vielzahl fachgruppenübergreifender allgemeiner und spezieller Gebührenordnungspositionen abgebildet.

Fazit: Die neue PFG soll die Grundversorgung stärken, bedient nicht alle Fachgruppen und wird zum Teil mit neuem Geld (126 Millionen $€$ für 2013) bezahlt

Dr. med. Heiner Pasch 